\title{
Risiko für Typ-1-Diabetes und Autoimmunthyreoiditis bei Zöliakie erhöht
}

Fragestellung: Wie hoch ist das Risiko von Kindern mit Zöliakie, an Typ-1-Diabetes oder Autoimmunthyreoiditis zu erkranken?

Hintergrund: Zöliakie ist eine systemische Autoimmunerkrankung, die bei etwa $1 \%$ der Bevölkerung auftritt. Sie verursacht primär eine Entzündung und Malabsorption im Dünndarm. Pathogenetisch ist eine Autoimmunreaktion gegen Transglutaminase bei genetisch empfänglichen Individuen, die häufig HLA-DQ2oder DQ8-Haplotypen aufweisen. Dieser genetische Hintergrund wird auch mit Typ-1-Diabetes und der Entwicklung von Autoimmunthyreoiditis in Verbindung gebracht. Daher wird bei beiden Erkrankungen ein Screening auf Zöliakie empfohlen. Bisher gibt es aber zum erhöhten Risiko anderer Autoimmunerkrankungen bei Zöliakie nur von Erwachsenen oder aus Cross-sectional-Datenbanken Material. Diese Studie liefert also die ersten populationsbezogenen Daten zum relativen Risiko für Typ-1-Diabetes und Autoimmunthyreoiditis bei Zöliakie im Kindesalter.

Patienten und Methoden: Die retrospektive Studie wurde in einer Region im Nordosten Italiens an einer Population von

\section{Originalie}

Canova C, Pitter G, Ludvigsson JF et al. Celiac disease and risk of autoimmune disorders: a population based matched birth cohort study. J Pediatr 2016. Mar 24 [Epub ahead of print] 1,2 Millionen Menschen gemacht. Insgesamt umfasste die Studie 213.635 Kinder und Jugendliche, die in den Jahren 1989-2011 dort geboren wurden. Es wurden auf Grundlage der Gesundheitsdatenbank Diagnosen und die Ergebnis- se sämtlicher pathologischen Berichte analysiert, zusätzlich bezog man Krankhausentlassungsberichte ein. Die für Zöliakie-, Diabetes- und Autoimmunthyreoiditis spezifischen ICD-Codes und Verordnungen von Insulin, Thyroxin und Thyreostatika kamen in die Auswertung, ebenso wie Fälle, bei denen vor der Zöliakie eine andere Autoimmunkrankheit auftgetreten war. Die Daten wurden einer alters- und geschlechtsgematchten Gruppe mit jeweils fünf Individuen pro Fall gegenübergestellt (1215 Zöliakiepatienten, 6075 Kontrollen.

Ergebnisse: 72\% der Zöliakien waren bioptisch gesichert. Insgesamt wurden 71 Typ-1-Diabetes-(T1D-)Patienten und $113 \mathrm{Au-}$ toimmunthyreoiditisfälle gefunden. Autoimmunhyperthyreose hatten nur sechs Patienten, daher wurden mit dieser niedrigen Zahl keine weiteren Analysen durchgeführt. Typ-1-Diabetes und Autoimmunthyreoiditis (AIT) hatten erwartungsgemäß eine höhere Prävalenz in der Zöliakiegruppe (T1D n=44; 3,6\%; AIT $\mathrm{n}=58 ; 4,8 \%$ ) als in der Kontrollgruppe (T1D $\mathrm{n}=27 ; 0,5 \%$; AIT $\mathrm{n}=55 ; 0,9 \%)$. Die Patienten waren jünger bei komorbider Zöliakie (T1DM 5 Jahre vs. 6 Jahre; AIT 11 Jahre vs. 15 Jahre). 0,7\% aller Zöliakiepatienten hatten mehr als eine Autoimmunerkrankung, versus $0,2 \%$ in der Kontrollgruppe. Das relative Risiko für T1D bei Zöliakie liegt bei 2,50 (gerade nicht signifikant), das für AIT bei 4,64. 3,1\% bekamen nach T1D noch eine Zöliakie (OR 12,66!).

Schlussfolgerungen: Kinder und Jugendliche mit Zöliakie haben ein erhöhtes Risiko für Autoimmunthyreoditis und zu einem gewissen Grad auch für Typ-1-Diabetes. Ein entsprechendes Antikörper-Screening ist daher offenbar sinnvoll.

\section{- Kommentar von PD Dr. med. Thomas M. Kapellen}

\section{Screening auf beide Erkrankungen bei Kindern mit Zöliakie sinnvoll}

Der Zusammenhang zwischen Zöliakie und anderen Autoimmunerkrankungen wurde sowohl für Erwachsene als auch Kinder wiederholt beschrieben, ohne bislang populationsbezogene Daten für Kinder zu kennen. Das macht diese Studienergebnisse so wichtig. Insgesamt gab es bislang auch nur wenige Daten zur Komorbidität von Autoimmunschilddrüsenerkrankungen und Zöliakie. Es zeigte sich ein Trend, wonach Patienten mit Zöliakie früher eine zweite Autoimmunerkrankung bekommen. Typischerweise tritt eine Autoimmunthyreoiditis (AIT) deutlich häufiger bei Mädchen auf. Zusammen mit einer Zöliakie ist das Risiko aber für Jungen deutlich erhöht (relatives Risiko 20,0!). Insgesamt könnten die Ergebnisse aber auch von Screeningempfehlungen zur AIT bei Zöliakie beeinflusst sein. Nicht auszuschließen sind einige übersehene Fälle - insbesondere von AIT -, wenn noch keine medikamentöse Behandlung erfolgt und bei latenter Hypo- thyreose eventuell auch die Diagnose noch nicht adäquat dokumentiert wird. Bislang beinhalten die Leitlinien pädiatrischer Gastroenterologen zur Zöliakie noch keine generelle Screeningempfehlung für AIT und T1D. Das sollte angesichts der vorliegenden Daten sicher überdacht werden.

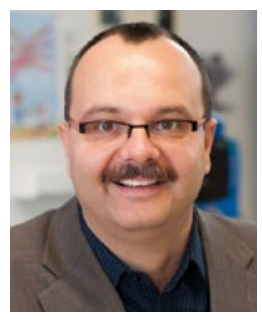

PD Dr. med. Thomas M. Kapellen

Universitätsklinik für Kinder und Jugendliche,

Frauen und Kinderzentrum

Liebigstr. 20a, 04103 Leipzig

ThomasMichael.Kapellen@medizin.

uni-leipzig.de 